\title{
Solving delay differential equations using coupled block method
}

\begin{abstract}
A variable step size variable order coupled block method for the numerical solution of delay differential equation is described. The approximation of the delay term is calculated using divided difference interpolation. The numerical results are presented and it shows that the proposed code is suitable for solving delay differential equations.
\end{abstract}

Keyword: Coupled block method; Delay differential equation 